\title{
Object Contour in Low Quality Medical Images in Curvelet Domain
}

\author{
Vo Thi Hong Tuyet \\ Faculty of Information Technology \\ Ho Chi Minh City Open University \\ Ho Chi Minh City, VietNam
}

\author{
Nguyen Thanh Binh ${ }^{*}$ \\ Faculty of Computer Science and Engineering \\ Ho Chi Minh City University of Technology, VNU-HCM \\ Ho Chi Minh City, Vietnam
}

\begin{abstract}
The diagnosis and treatment are very important for extending the life of patients. The small abnormalities may also be manifestations of the diseases. One of the abnormalities is the contour of each object in medical images. Therefore, the contour is very important and special to low quality medical images. In this paper, we propose a new method to detect the object contour of low quality medical images based on the self affine snake, one of the types of active contour models. The method includes two periods. Firstly, we use augmented lagrangian method to remove noise and detect edges in low quality medical images in curvelet domain. Finally, the active contour model is improved to show the contour of objects. After comparing the appearance of the contour and the time processing with other algorithms, we confirm that the proposed method is better.
\end{abstract}

Keywords-Object contour; curvelet transform; augmented lagrangian

\section{INTRODUCTION}

Nowadays, many diseases cannot be detected by the naked eye, such as the diseases of bone, liver, cancer, etc. If dangerous diseases are detected early, the diagnosis and the treatment will be easier. Therefore, the medical image is an important tool for specialists. They contain much important information inside human body, and the contour of each object is very necessary. The location of boundary is sometimes to refer to as fracture, enlargement or tumor. Most of the important task in medical image is edge detection. The result of edge detection depends on the quality of the image [2], [5]. However, the quality of medical images depends on many factors such as: technical skills, machines, printer, etc. because these reasons make medical images have noise or blur details, and the quality of them is reduced. So, this is a big challenge.

Medical images are abundant about types. So, the boundary of each agency is difficult for detection because of the intensity of colorful or connection between them. In the last time, there were many methods for object contour in natural image, such as Sobel [4], B-spline [6]-[10] or the generation types of wavelet transform as [1], [6], [11], [12]. Brigger and his partners used B-spline snakes to detect the boundaries of objects in [7], multiscale of them, and to develop it as a flexible tool in [10]. Then, [23] is a method which also uses snake for detection and segmentation in medical images. The combination between curvelet with selfaffine mapping is proposed in [25] for the medical images which have low quality. So, the relation between transform and snake can be useful for contour detection. If the results from edge detection step are good, the contour will be more accurate and clearer.

Although multi-scale or space domain gives positive results, the wavelet transform is one of the methods that is space domain. Discrete wavelets transform (DWT) [13] and complex wavelet [12] is continued. However, DWT has three disadvantages [14]: poor directionality, shift sensitivity, lack of phase information. So, the emergence of more optimal methods restored, such as: contourlet transform [15], nonsubsampled contourlet transform [16], [17], ridgelet transform or curvelet transform [18] and shearlet transform in [1].

Medical images contain one or more objects. They have noised or blurred pixels or both, and called as weak object. A concept of strong and weak objects in contour is defined in [12]. Therefore, the low quality is the big challenge for object contour detection. In the recent years, the applied of curvelet threshold in medical image is very powerful such as denoising [26], detection [27] and segmentation [28]. However, the time processing for using curvelet coefficients is very high. Yet, the slow time can be accepted in medicine as long as the result is good. However, the time can be smaller, the life can be longer. With this problem, augmented lagrangian method [3] is a good resolution.

Based on the previous knowledge, we use augmented lagrangian method (ALM) and selft-affine snake to detect the boundary of medical images. However, we use the space domain, curvelet domain, instead of the frequency domain. The contributions of this paper are: 1) to design a competent curvelet domain adaptive threshold, capable to increment the quality of medical image. 2) The active contour model is explained. 3) Authors proposed a new method for object contour in low quality medical image in curvelet domain. The rest of the paper is organized as follows: In Section 2, we present the basic concepts which relates to the proposed method in this paper, the Section 3 present the proposed method for object contour, the Section 4 present the experiments and results. The final section is conclusions. 


\section{BRIEF KNOWLEDGE}

\section{A. Curvelet Transform}

Wavelet transform is a popular method for image restoration [14]. Developing it, the new generation wavelet is showed; and the curvelet is one of them. The first generation of curvelet transform is ridgelet transform [18], [19]. In ridgelet domain, the hard threshold and soft threshold are applied. If we call $\mathrm{T}_{\text {hard }}$ to be the hard threshold and $\mathrm{T}_{\text {soft }}$ to be the soft threshold, $\lambda \geq 0$ is parameter wavelet, $I$ is normal parameter value. We have equations:

$$
\mathrm{T}_{\text {hard }}\left(\hat{d}_{j k}, \lambda\right)=\hat{d}_{j k} I\left(\left|\hat{d}_{j k}\right|>\lambda\right)
$$

and

$$
\mathrm{T}_{\text {soft }}\left(\hat{d}_{j k}, \lambda\right)=\operatorname{sign}\left(\hat{d}_{j k}\right) \max \left(0,\left|\hat{d}_{j k}\right|-\lambda\right)
$$

Ridgelet transform can be adapted to represent objects with curved edges that are almost straight [21]. Two concepts are monoscale and multiscale, which represent the theory of ridgelets [25] based on the ridge functions to develop ridgelet system. The orthonormal ridgelets are indexed using as (3):

$$
\lambda=(s, l, a, a l, x)
$$

where, $\mathrm{s}$ is the ridge scale, 1 is the ridge location, $\mathrm{a}$ is the angular scale, al is the angular location and $\mathrm{x}$ is a gender token. The ridgelet transformation can be showed as follows [20]:

- Smoothing step based on the Fourier transform.

- Calculating bivariate ridgelet and depended on the radon transform that is the important process for ridgelet values.

- $\quad$ Performing the scale wavelet transform with ridgelet coefficients to define the result images.

The curvelet transform is a non-adaptive technique. It is the representation of multi-scale of objects. The curvelet is superior method to keep information and to improve the quality of images which have curves between edges. Similar to wavelets, curvelet transform can be dilated in two dimensions and translated. And like ridgelets, curvelets can be applied for all scales, locations and orientations. Each subband of curvelet is a curve with width $\approx$ length $^{2}$ and is analyzed by a local ridgelets. The subbands of curvelets have the nonstandard form $\left[2^{2 \mathrm{~s}}, 2^{2 \mathrm{~s}+2}\right]$. Authors in [18], [19] give the process of curvelet transform to include four steps:

Firstly, the subband is decomposed. The image $f$ is decomposed into subbands by [18], [19]:

$$
f \mapsto\left(P_{0} f, \Delta_{1} f, \Delta_{2} f, \ldots\right)
$$

Each subband is the presentation of the value which needs to adapt to reconstruct coefficients.
Secondly, smooth partitioning. Each subband is applied with scale which has the sidelength $\sim 2^{-s}$, as follows by (5):

$$
\Delta_{s} f \mapsto\left(w_{Q} \Delta_{s} f\right)_{Q \in Q_{S}}
$$

where, $w_{Q}$ is a collection of smooth window localized around dyadic squares:

$$
Q=\left[k_{1} / 2^{s},\left(k_{1}+1\right) / 2^{s}\right] \times\left[k_{2} / 2^{s},\left(k_{2}+1\right) / 2^{s}\right]
$$

Thirdly, the renormalization. Each resulting square is renormalized to unit scale

$$
g_{Q}=\left(T_{Q}\right)^{-1}\left(w_{Q} \Delta_{S} f\right), \quad Q \in Q_{S}
$$

Finally, ridgelet analysis. Each square is analyzed via the discrete ridgelet transform.

While restore the images, besides of the curvelet coefficients, we can use the threshold and filter [14], [21] to support or combine between them [20]-[22]. But the execution time is very long. The time is slow because the adapting of the image to many filters or thresholds of transforms.

\section{B. Augmented Lagrangian Method}

When restoring the image restoration, any method must recover the sharpness of the image. The idea to minimize a total variation optimization problem for spatial temporal data was proposed [3]. It is Augmented Lagrangian Method (ALM) to solve the constrained problem. When we call a vector denoting the unknown (potentially sharp) image which has size $\mathrm{Mx} \mathrm{N}$ is called $\mathrm{f}$ ( $\mathrm{f} \in R^{M N \times 1}$ ), where $\mathrm{f}$ is an ingredient of equation to find the observed image $\mathrm{g}, \mathrm{g} \in R^{M N \times 1}$. It is a linear shift invariant imaging system to calculate as: $\mathrm{g}=\mathrm{Hf}+\eta$, where $\mathrm{f}$ is a vector denoting the unknown (potentially sharp) image of size $\mathrm{Mx} \mathrm{N}, \mathrm{g}$ is a vector denoting the observed image, $\eta \in R^{M N \times 1}$ is a vector denoting the noise/blur, and the matrix $\mathrm{H} \in R^{M N \times M N}$ is a linear transformation representing convolution operation. This algorithm includes two types: TV/L1 minimization (for denoising image) and TV/L2 minimization (for deblurring image). They were defined as [3]:

$$
\underset{\mathrm{f}}{\operatorname{minimize}} \frac{\mu}{2}\|H f-g\|^{2}+\|f\|_{T V}
$$

and

$\underset{\mathrm{f}}{\operatorname{minimize}} \mu\|H f-g\|_{1}+\|f\|_{T V}$

where, $\mu$ is the regularization parameter. The authors were to find a saddle point of $\mathrm{L}(\mathrm{f}, \mathrm{u}, \mathrm{y})$. Then, they used the alternating direction method (ADM) to solve f-subproblem, $\mathrm{u}$ subproblem with TV/L2 and f-subproblem, u-subproblem and r-subproblem with TV/L1. The equation as [3]:

$$
\underset{\mathrm{f}, \mathrm{u}}{\operatorname{minimize}} \frac{\mu}{2}\|\mathrm{Hf}-\mathrm{g}\|^{2}+\|\mathrm{u}\|_{1}
$$


and

$$
\underset{\mathrm{f}, \mathrm{r}, \mathrm{u}}{\operatorname{minimize}} \mu\|r\|_{1}+\|\mathrm{u}\|_{1}
$$

Subject to $\mathrm{r}=\mathrm{Hf}-\mathrm{g}$ and $\mathrm{u}=$ Df. Augmented lagrangian method can be summarized as follows [3]:

- Input: the information of vector denoting, convolution matrix, regularization parameter, the isotropic total variation.

- $\quad$ Set parameter with value default. This step depends on other types of denoising or deblurring.

- Initialize for the first value of each problem.

- Compute the matrices of displacement in directions, such as: horizontal, vertical and temporal.

- Check this value is coverage or not. If true, ALM will remove values (noise or blur) and update parameters. Then the checking process is continued.

- When having any value to be not coverage, the algorithm will stop to check.

\section{Active Contour Model}

Active contour model, snake, is a method to detect boundaries of object in images. The position of a snake parametrically is known by $\mathrm{v}(\mathrm{s})=(\mathrm{x}(\mathrm{s}), \mathrm{y}(\mathrm{s}))$ and energy functional as [7], [10]:

$$
E_{\text {snake }}^{*}=\int_{0}^{1} E_{\text {snake }}(v(s)) d s=\int_{0}^{1} E_{\text {int }}(v(s))+E_{\text {image }}(v(s))+E_{\text {con }}(v(s)) d s
$$

where, $\mathrm{E}_{\text {int }}$ represents the internal energy of spline due to bending is:

$$
E_{\text {int }}=\left(\alpha(s)\left|v_{s}(s)\right|^{2}+\beta(s)\left|v_{s s}(s)\right|^{2}\right) / 2
$$

$\mathrm{E}_{\text {image }}$ gives rise to the image forces, and $\mathrm{E}_{\text {con }}$ gives rise to the external constraint forces to calculate by [24]:

$$
E_{c o n}=\frac{1}{2}\left(\alpha(s)\left|v_{s}(s)\right|^{2}\right)
$$

where, $\alpha(s)$ and $\beta(s)$ are user-defined weights. The $\alpha(s)$ is a large weight for the continuity term penalizes. The distance between points in the contour is changed by $\alpha(s)$. In addition, $\beta(s)$ is for the smoothing term of the contour. The features of images are the showing of image energy. These features are calculated by [24]:

$$
E_{\text {image }}=w_{\text {line }} E_{\text {line }}+w_{\text {edge }} E_{\text {edge }}+w_{\text {term }} E_{\text {term }}
$$

where, $\mathrm{w}_{\text {line }}, \mathrm{w}_{\text {edge }}, \mathrm{w}_{\text {term }}$ are weights of these salient features. The process of snake includes computing force and then combining them and converging snake model. With selfaffine snake includes a step in the first of process which is extracting self-affine maps. However, the active contour model is only to apply when we have the object detection.

\section{OBJECT CONTOURS IN CURVELET DOMAIN}

Energy reducing is a popular direction for presenting the contour methods. In [22], the authors use energy reducing, self-affine snake in wavelet domain for contour detection. Edge detection is almost difficult because of the quality of images. The result of the contour object detection depends on the results of the edge detection step. Special with medical images, the quality of them is bad because of many reasons: skill of technical, machine, etc. ... Edge detection is hard work because it is easy to lose information in medical images. Curvelet is one of the best choices for denoising medical images. In [24], [25], the authors use curvelet domain and Snake for contour detection and segmentation which have positive results. So, we can develop from it.

Based on these ideas, we propose a new method for object contour. The process method is presented clearly in Fig. 1. In Fig. 1, the process method includes two periods. Firstly, object detection based on ALM for smoothing. Finally, the self-affine Snake for active contour. Both period 1 and 2 are also in curvelet domain.

\section{A. Object Detection based on ALM for Smoothing}

Firstly, the input of the method is low quality medical images. These images have blur or noise values. So, objects in them are weak. Then, the decomposition which level is 5 and direction is 32 to be applied. The curvelet's process is as follows: decompose input images into curvelet domain with scales depending on the level and direction. Then, the loop statement will begin in 1 . Each repeat, calls block size, applies digital ridgelet transform.

Secondly, the smoothing image: this step applies TV/L1 process of ALM, which follows [3]:

$$
\text { minimize }_{f, r, u} \mu\|r\|_{1}+\|u\|_{1}
$$

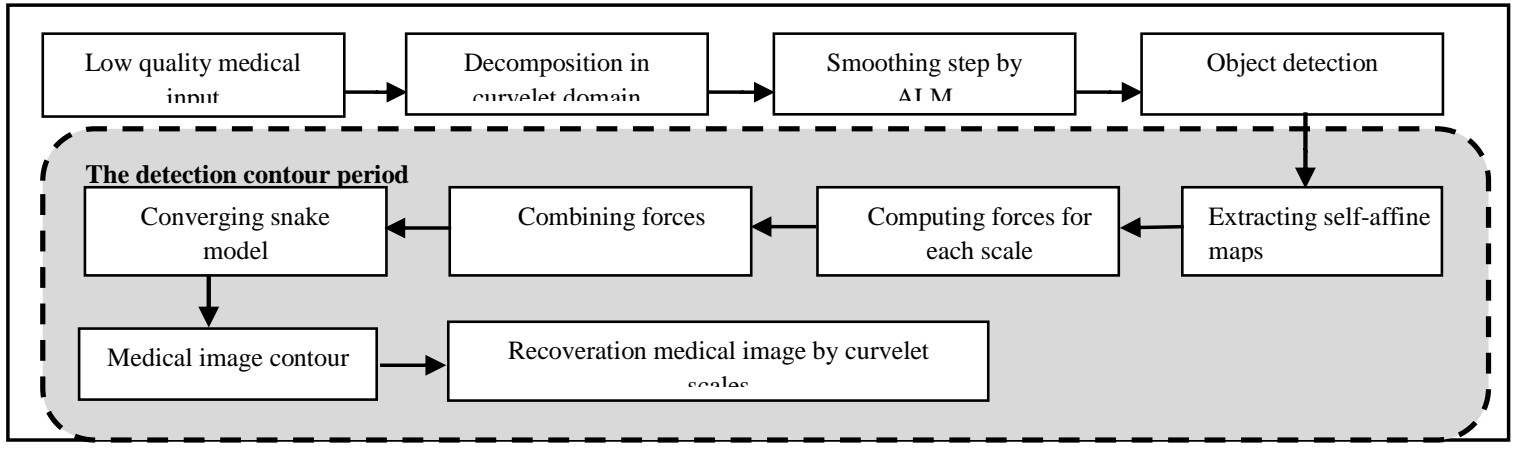

Fig. 1. The proposed method for object contour. 
Equation (16) is solved as follows:

INPUT: vector denoting the observed image $(\mathrm{g})$ and convolution matrix $(\mathrm{H})$, regularization parameter $\mu$, the isotropic total variation $\beta_{x}, \beta_{y}, \beta_{t}$.

OUTPUT: the medical image, which is denoised and deblurred. The object is to apply smoothing.

BEGIN:

- Set parameter with value default for $\rho_{r}=2, \rho_{0}=100$ where $\alpha_{0}=0.7$ and $\rho_{r}$ : regularization parameter.

- Initialize $\mathrm{f}_{0}=\mathrm{g}, \mathrm{u}_{0}=\mathrm{Df}_{0}, \mathrm{y}_{0}=0, \mathrm{r}_{0}=\mathrm{Hf}_{0}-\mathrm{g}, \mathrm{z}_{0}=0$

- Compute the matrices of the first-order forward finite difference operators along the horizontal, vertical and temporal directions.

- While (not coverage) do:

(i) Solve the f-subproblem is:

$$
\text { minimize }_{f}=\frac{\rho_{0}}{2}\|r-H f+g\|^{2}+\frac{\rho_{r}}{2}\|u-D f\|^{2}+z^{T} H f+y^{T} D
$$

where, $D=\left[\begin{array}{lll}D_{x}^{T} & D_{y}^{T} & D_{t}^{T}\end{array}\right]^{T}$ with: $\mathrm{D}_{\mathrm{x}}$, is the order forward finite-difference operators along the horizontal direction, $\mathrm{D}_{\mathrm{y}}$ is vertical direction and $\mathrm{D}_{\mathrm{t}}$ is temporal direction.

f-subprobem is improved by equation:

$$
f=\mathcal{F}^{-1}\left[\frac{\mathcal{F}\left[\rho_{0} H^{T} g+H^{T}\left(\rho_{0} r-z\right)+D^{T}\left(\rho_{r} u-y\right)\right]}{\rho_{0}|\mathcal{F}[H]|^{2}+\rho_{r}\left(\left|\mathcal{F}\left[D_{x}\right]\right|^{2}+\left|\mathcal{F}\left[D_{y}\right]\right|^{2}+\left|\mathcal{F}\left[D_{t}\right]\right|^{2}\right)}\right]
$$

where, $\mathcal{F}$ denotes the $3 \mathrm{D}$ Fourier Transform operator.

(ii) Solve u-subproblem:

$$
\begin{aligned}
& u_{k+1}=\underset{u}{\arg \min }\|u\|_{1}-y_{k}^{T}\left(u-D f_{k+1}\right)+\frac{\rho_{r}}{2}\left\|u-D f_{k+1}\right\|^{2} \\
& \text { where, } \quad u_{x}=\max \left\{\left|v_{x}\right|-\frac{1}{\rho_{r}}, 0\right\} * \operatorname{sign}\left(v_{x}\right)
\end{aligned}
$$

(iii) Solve r-subproblem:

minimize $_{r} \quad \mu\|r\|_{1}-z^{T} r+\frac{\rho_{0}}{2}\|r-H f+g\|^{2}$

by equation: $r=\max \left\{\left|H f-g+\frac{1}{\rho_{0}} z\right|-\frac{\mu}{\rho_{0}}, 0\right\} * \operatorname{sign}\left(H f-g+\frac{1}{\rho_{0}} z\right)$

(iv) Update the Lagrange multiplier y and $z$ :

$y_{k+1}=y_{k}-\rho_{r}\left(u_{k+1}-D f_{k+1}\right)$

$z_{k+1}=z_{k}-\rho_{0}\left(r_{k+1}-H f_{k+1}+g\right)$

(v) Update:

$$
\rho_{r}=\left\{\begin{array}{l}
\gamma \rho_{r}, \text { if }\left\|u_{k+1}-D f_{k+1}\right\|_{2} \geq \alpha\left\|u_{k}-D f_{k}\right\|_{2} \\
\rho_{r}, \text { otherwise }
\end{array}\right.
$$

(vi) Check convergence: if $\left\|f_{k+1}-f_{k}\right\|_{2} /\left\|f_{k}\right\|_{2} \leq$ tol then break

\section{End while.}

END

Thirdly, the object detection. In contour detection, after smoothing step, methods begin to calculate the strengths between edges together known to be the determining gradients. We use the equation of Eucidean distance as (26):

$$
E=\left(\sum_{i=1}^{n}\left|x_{i}-y_{i}\right|^{2}\right)^{1 / 2}
$$

where, $(\mathrm{x}, \mathrm{y})$ is coordinates of image pixels. E will be used to define edges to be shown. Each pixel in the gradient image, $45^{\circ}$ for direction and the connected neighborhoods help for the calculating process. The next problem is to show the relationships between pixels by direction, it is:

$$
\theta=\arctan \left(\frac{\left|G_{y}\right|}{\left|G_{x}\right|}\right)
$$

where, $G_{x}$ and $G_{y}$ are the gradients in the $x$ and $y$ directions respectively. $\mathrm{G}_{\mathrm{x}}$ and $\mathrm{G}_{\mathrm{y}}$ are as (26).
The edges are connected when the strength is greater than 8 -connected neighborhoods, and it will be removed. The boundaries of medical images are given, but they are not clear and powerful. We continue with them in the next period.

\section{B. Contour Detection}

In this period, we show the boundaries from the edges to be detected of the first period. The processing of this period, we present [24] as: extracting the self-affine maps, combining forces, converging snake model. In here, we don't use the coefficients of curvelet as [24]. We improve the number of edges in the part $\mathrm{A}$ of this section. The matching code $\mathrm{C}$ is also evaluated by (28):

$$
C=\sum_{x \in M_{i}}\left|g(x)=g\left(m_{i}(x)\right)\right|
$$

where, $\mathrm{g}(\mathrm{x})$ is the intensity values. This is a first step of our methods. The second step is decomposition in Gaussian steps and the sum calculating (13) and (14). From this, the 
energy of medical images has given, similar to (15). This is the grounds for forces.

Then, the coefficients for showing the boundaries of objects must fit with:

$$
2^{\mathrm{n}} \times \mathrm{E}_{\text {image }}>\mathrm{E} \text { and } \mathrm{n}>\log _{2}\left(\mathrm{E} / \mathrm{E}_{\text {image }}\right)
$$

where, $\mathrm{E}$ is the values of equation (15) and $\mathrm{n}$ is the coefficients of smoothing step (by the comparison the smaller value between ALM and curvelet). If any value fits, it will be used for restoration and appearance. If not, it will not be a member of object contour. The difference of our method with [24] is we don't only depend on coefficients of denoising images and improve this result of contour. Because of the denoising in space domain can lack information of medical images. We want to keep edges and connect between weak edges. They have very little gray intensity.

\section{EXPERIMENTS AND RESULTS}

The contour of objects in medical images has been very important because the size and location of them is manifestation of a disease. In Section 3, we present our method for object detection and improve the quality of boundaries in medical images. In the proposed method, we do not only use the features of curvelet transform for denoising medical images as other methods [24], [25] but also apply

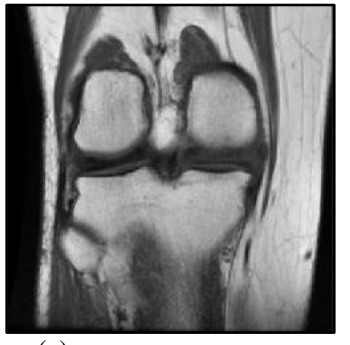

(a)

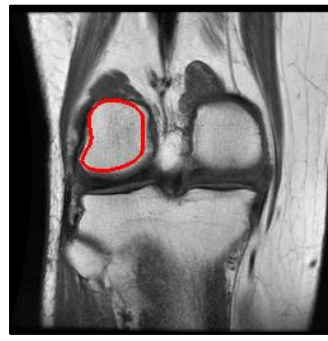

(b)

ALM for smoothing images. That is the preparatory step for the object detection in curvelet domain. Then, the self-affine snake in curvelet domain show the contour of objects. But we have another improvement to be the comparative values of forces.

The results of our proposed method are compared with the other methods such as self-affine snake [23] and curveletbased geodesic snake method [25]. We have more edges and clear contour than other methods. We collect dataset from many hospitals with many sizes in more than 100 types of medical images. We test and show the contour of objects about the clarity and accuracy. Besides, we also compare the time processing between methods.

Fig. 2 is the results of the proposed method and other methods with low quality medical images. Fig. 2(a) is the low quality medical images, Fig. 2(b) is the result of self- affine snake [23], Fig .2(c) is the result of curvelet-based geodesic snake method [25] and the proposed method result is the Fig. 2(d).

Fig. 3 is another example about testing. Similar to Fig. 2, Fig. 3(a) is the low quality medical images. Fig. 3(b) is the result of self-affine snake [23]. Fig. 3(c) is the result of curvelet-based geodesic snake method [25] and the proposed method result is Fig. 3(d).

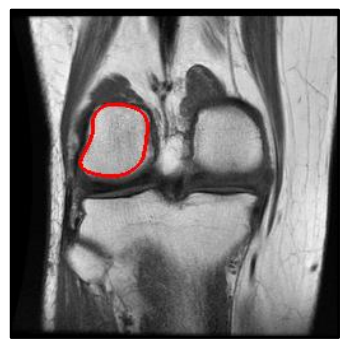

(c)

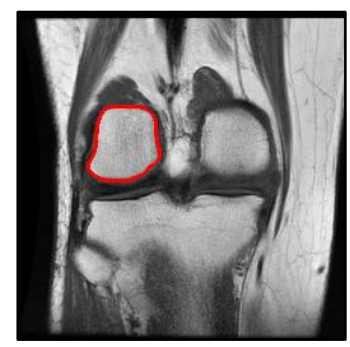

(d)

Fig. 2. The results of contour detection by other methods with weak object: (a) The weak object in a noised and blurred medical image. (b) The result of detection by self-affine snake [23] with the time processing $~ 3.152$ seconds. (c) The result of detection by curvelet-based geodesic snake method [25] with the time processing $\sim 4.523$ seconds. (d) The result of detection by the proposed method with the time processing $\sim 4.047$ seconds.

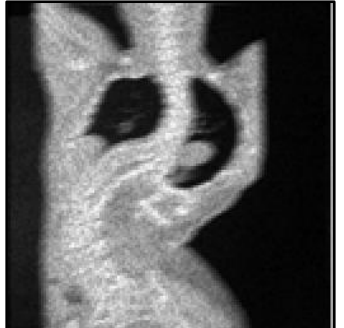

(a)

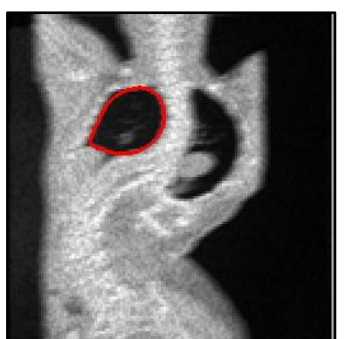

(b)

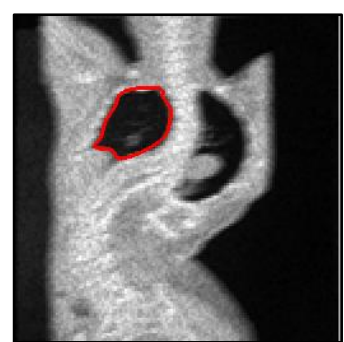

(c)

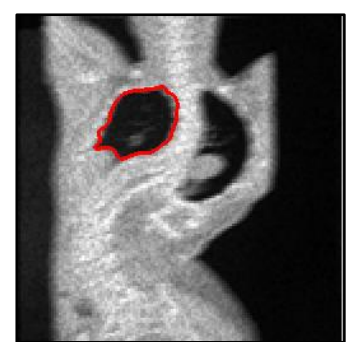

(d)

Fig. 3. The results of contour detection by other methods with weak object. (a) The weak object in a noised and blurred medical image. (b) The result of detection by self-affine snake [23] with the time processing 3.941 seconds. (c) The result of detection by curvelet-based geodesic snake method [25] with the time processing $\sim 5.067$ seconds. (d) The result of detection by the proposed method with the time processing $\sim 4.797$ seconds.

The images in Fig. 2(a) and 3(a) also have no powerful objects. Because of the addition of 0.0005 of noise value and 0.001 of blur value in them. These numbers are popular in images.

From Fig. 2 and 3, we can see the accuracy of contour detection by our method is better. The time processing is smaller than curvelet-based geodesic snake method [25].
Because the proposed method uses ALM to improve the smoothing step to shorten the time processing. The time processing of self-affine snake [23] is the shortest, but the accuracy of contour is not good. Our results are better than other methods because of two reasons:

Firstly, on one hand, that is improving of the smoothing step by ALM and using curvelet coefficients to active contour. 
In edge detection step, the time processing is shortened to base on the ALM that removes noise from input images, because it does not depend on any threshold or filter. This is different from curvelet-based geodesic snake method [25]. The calculating of curvelet coefficients is applied in the next step, after denoising in smoothing step. That is more accurate and faster than other method. With medical images, the small details are very useful. We choose ALM to improve the smoothing step because of this reason. We do not want to lose the any pixels or weak values from input images. Because any pixel has a difference from others, it may be an early express.

Secondly, on the other hand, the curvelet coefficient is very useful for active contour. The combining forces need the wavelet scales. In curvelet domain, these coefficients are very fit in this step. Moreover, the scales and the number of direction are in decomposition. Each subband of curvelet is a curve, to medical images that is an advantage. The previous step, edge detection, has good results and keeps much information of input images; the object contour is an advantage to be showed. And specially, we still use the results from the comparision with ALM coefficients to show forces.

\section{CONCLUSIONS AND FUTURE WORKS}

Object contour is an important task for segmentation, but the edge detection decides the results of contour. So, the improving result in the first step is necessary. When smoothing by transform, the result is very good but the processing time is very slow. We use the ALM to shorten the time but still keep information and applying self-affine snake to active contour. All of the steps are in curvelet domain, but we don't only base on the curvelet coefficients. The authors compare the results with self-affine snake [23] and curveletbased geodesic snake method [25]. The comparison is not only the sharp contour, but also the execution time of algorithms in low quality medical images. In the future works, we extend to strong low quality medical images. We calculate and compare the information in medical images before and after applying our method.

\section{ACKNOWLEDGMENT}

This research is funded by Vietnam National University HoChiMinh City (VNU-HCM) under grant number C201720-20. The authors thank the anonymous reviewers for their constructive comments, which improved the quality of the paper.

\section{REFERENCES}

[1] G. Easley; D. Labate; W.Q. Lim. "Sparse directional image representations using the discrete shearlet transform". Journal of Applied and Computational Harmonic Analysis, Vol 25, pp. 25-46, 2008.

[2] L. Zhai, S. Dong; H. Ma. "Recent methods and applications on image edge detection". International Workshop on Geoscience and Remote Sensing, pp. 332-335, 2008.

[3] Stanley H. chan; Ramsin Khoshabeh; Kristofor B. Gibson; Philip E. Gill and Truong Q. Nguyen. "An Augmented Lagrangian Method for Total Variation Video Restoration”. IEEE Trans. Image Process, Vol 20 (11), pp. 3097-3111, 2011.

[4] O. R. Vincent; O. Folorunso. "A Descriptive Algorithm for Sobel Image Edge Detection". Proceedings of Informing Science \& IT Education Conference, pp. 97-107, 2009.
[5] Bryan S. Morse. "Edge Detection. Brigham Young University". http://homepages.inf.ed.ac.uk, (last accessed: 28 Nov 2017)

[6] Wang Yuping; Cai Yuanlong. "Multiscale B-spline wavelet for edge detection”. Science in China (Series A), Vol. 38(4), pp. 499-512, 1995.

[7] Patrick Brigger; Michael Unser. "Multi-scale B-spline Snakes for General Contour Detection". Wavelet Applications in Signal and Image Processing VI, SPIE, Vol 3458, pp. 92-102, 1998.

[8] A.D. Bhatt; R.V. Warkhedkar. "Reverse engineering of human body: a B-Spline based heterogeneous modeling approach". Computer-Aided Design and Applications, Vol 5, pp. 194-208, 2008.

[9] A.D. Bhatt; R.V. Warkhedkar. "Material-solid modeling of human body: a heterogeneous B-Spline based approach". Computer-Aided Design, Vol. 41, pp. 586-597, 2009.

[10] P. Brigger; J. Hoeg; M. Unser. "B-Spline snakes: a flexible tool for parametric contour detection". IEEE Transactions on Image Processing, Vol. 9, No. 9, pp. 1484-1496, 2000.

[11] Lei Zhang; Paul Bao. "Edge detection by scale multiplication in wavelet domain". Pattern Recognition Letters 23, pp.1771-1784, 2002.

[12] Nguyen Thanh Binh. "Image contour based on context aware in complex wavelet domain". Journal of Human-centric Computing and Information Sciences, Vol 5(14), pp. 1-17, 2015.

[13] Marcin Kociolek; Andrzej Materka; Michal Strzelecki; Piotr Szczypínski. "Discrete Wavelet transform - derived features for digital image texture analysis". Proc. of International Conference on Signals and Electronic Systems, pp. 163-168, 2001.

[14] Nguyen Thanh Binh; Ashish Khare. "Image Denoising, Deblurring and Object Tracking, A new Generation wavelet based approach". LAP LAMBERT Academic Publishing, 2013.

[15] Minh N. Do; Martin Vetterli. "The contourlet transform: an efficient directional multiresolution image representation". IEEE Trans. Img. Processing, pp. 2091-2106, 2005.

[16] Arthur L. da Cunha; Jianping Zhou; Minh N. Do. "Nonsubsampled Contourlet Transform: Theory, Design, and Applications". IEEE Trans. Img. Proc, pp. 3089-3101, 2005.

[17] Arthur L. da Cunha; J. Zhou; Minh N. Do. "Nonsubsampled Contourlet Transform: Filter design and applications in denoising". Proceedings of the 12th IEEE International Conference on Image Processing, pp. 749$752,2005$.

[18] Zhang; J. M. Fadili; J. L. Starck. "Wavelets, ridgelets and curvelets for poisson noise removal". IEEE Transactions on Image Processing, pp.1093-1108, 2008.

[19] Starck J L; Candès E J; Donoho D L. "The curvelet transform for image denoising”. IEEE Trans. Image Processing, Vol. 11(6), pp. 670-684, 2002.

[20] Nguyen Thanh Binh, Ashish Khare. "Multilevel threshold based image denoising in curvelet domain". Journal of computer science and technology, Vol 25, pp. 632-640, 2010.

[21] Nguyen Thanh Binh, Vo Thi Hong Tuyet. "The combination of adaptive filters to improve the quality of medical images in new wavelet domain", chapter 3 in book: Classification and Clustering in Biomedical Signal Processing, IGI Global Publication, pp 46-76, 2016.

[22] M. Saadatmand-Tarzjan; H. Ghassemian. "Self-affine snake: a new parametric active contour". IEEE Int. Conf. Signal Process. Commun, pp. 492-495, 2007.

[23] M. Saadatmand-Tarzjan; H. Ghassemian. "Self-affine snake for medical image segmentation". Pattern Recognition. Letters 59, pp. 1-10, 2015.

[24] Vo Thi Hong Tuyet, "Active contour based on curvelet domain in medical images", Proceedings of The International Conference on Nature of Computation and Communication, Vol 168, pp 325-333, 2016.

[25] Hao Shan; Jianwei Ma. "Curvelet-based geodesic snakes for image segmentation with multiple objects". Journal Pattern Recognition Letters, Vol. 31, pp. 355-360, 2010.

[26] Amir Moslemi; Amir Movafeghi; Shahab Moradi. CT Medical "Images Denoising Based on New Wavelet Thresholding Compared with Curvelet and Contourlet". International Journal of Computer, Electrical, Automation, Control and Information Engineering, Vol 9(10), pp. 21742179, 2015. 
[27] Jiange Liu; K. Andrea Scott; Ahmed Gawish; Paul Fieguth. "Automatic Detection of the Ice Edge in SAR Imagery Using Curvelet Transform and Active Contour", Journal of Remote Sens, Vol 8, pp. 480-489, 2016.
[28] Latha Subbiah; Dhanalakshmi Samiappan; P. Muthu. "Automated denoised ultrasound carotid artery image segmentation using curvelet threshold decomposition". IEEE International Conference on Wireless Communications, Signal Processing and Networking, pp. 2261-2266, 2017. 